\title{
15.
}

\section{Démonstration d'un théorème de Mr. Slonimsky sur les nombres, avec une application de ce théorème au calcul de chiffres.}

(Par l'édileur.)

Mr. Slonimsky de Bialystock en Pologne, homme de leltres distingué, auteur d'un cours des mathématiques, écrit en hébreu etc., communiqua, en Juillet 1844, à l'éditenr de ce journal (sans démonstration) un théorème très curieux, sur lequel il avait fondé une de ses ingénieuses machines à calculer. Ces machines furent alors presentées par lui à l'académie des sciences de Berlin; elles lui valurent ici une récompense; depuis elles ont remporté le second prix Demidof à l'académie des sciences de St. Pétersbourg, et il en publiera sous peu la déscription en langue russe.

L'éditeur de ce journal trouva une démonstration du théorème, qu'il communiqua à Mr. Slonimsky, et il l'aurait publiée plus tôt, s'il n'avait pas dû altendre la publication des machines que l'inventeur avait l'intention de faire. Plus tard il trouva encore une application du théorème an calcul de chiffres, nommément à la multiplication des chiffres, qui sous certaines conditions pent la faciliter considérablement et augmenter la sûrété des résultats.

Maintenant, autorisé par Mr. Slonimsky, à publier le théorème et sa démonstration, il les présente ici, avec l'application mentionnée.

\section{Théorèine.}

Soit $\boldsymbol{Z}$ un nombre quelconque, et désignons les chiffres de ce nombre, en allant de la droite vers la gauche, par $z_{1}, z_{2}, z_{3}, \ldots z_{\varepsilon}, \ldots$ Si sous le nombre $\boldsymbol{Z}$ on écrit ses multiples $2 \mathbf{Z}, 3 \mathbf{Z}, 4 \mathbf{Z}, 5 \mathbf{Z}, 6 \mathbf{Z}, 7 \mathbf{Z}, 8 \mathbf{Z}$ et $9 \boldsymbol{Z}$, de sorte que les unités, les dixaines, les centaines etc. soient en lignes verticales, il est clair, que la dernière ligne verticale, celle qui passe par le dernier chiffre $z_{1}$ de $Z$, contiendra les seconds chiffres des produits $2 z_{1}$, $3 z_{1}, 4 z_{1}, 5 z_{1}, 6 z_{1}, 7 z_{1}, 8 z_{1}$ et $9 z_{1}$. Mais il n'en sera pas ainsi de toute autre ligne verticale. Celle, par ex., qui passe par $z_{\epsilon}$, ne contiendra pas la 
série des seconds chiffres des produits $2 z_{\varepsilon}, 3 z_{\varepsilon}, 4 z_{\varepsilon}, 5 z_{\varepsilon}, 6 z_{\varepsilon}, 7 z_{\varepsilon}, 8 z_{\varepsilon}$ et $9 z_{\varepsilon}$. Pour obtenir les chiffres de cette ligne, on devra ajouter à la série des seconds chiffres des multiples de $z_{\varepsilon}$ une série complémentaire, qui dépendra des chiffres qui suivent $\boldsymbol{z}_{e}$ dans le nombre $\boldsymbol{Z}$. Or, quels que soient ces derniers chiffres, il n'existe que Vingt-huit séries complémentaires différentes, et en ajoutant à la série des seconds chiffres des multiples de $z_{\varepsilon}$, celle des 28 séries complémentaires qui convient aux chiffres qui dans $\boldsymbol{Z}$ suivent $\approx_{\varepsilon}$, on aura les chiffres de la ligne verticale qui passe par $z_{\varepsilon}$.

\section{Démonstration.}

A. Considérons les unités du chiffre $z_{\varepsilon}$ comme unités absolues, les chiffres qui suivent $\boldsymbol{z}_{\varepsilon}$ dans le nombre $\boldsymbol{Z}$ exprimeront alors une fraction décimale, toujours moindre que 1 , dont nous désignerons par $x_{\varepsilon-1}$ la valeur, et les séries complémentaires, desquelles parle le théorème, seront celles des entiers contenus dans $2 x_{\varepsilon-1}, 3 x_{\varepsilon-1}, 4 x_{\varepsilon-1}, 5 x_{\varepsilon-1}, 6 x_{\varepsilon-1}, 7 x_{\varepsilon-1}, 8 x_{\varepsilon-1}$ et $9 \boldsymbol{x}_{\varepsilon-1}$.

B. Supposons maintenant que la fonction $x_{\varepsilon-1}$ aille en croissant par degrés insensibles, depuis zéro jusqu'à 1 , il est clair que chaquefois que la fraction $x_{\varepsilon-1}$ passera par une fraction irréductible $\frac{m}{n}$, dont le dénominateur $n$ est un des nombres $2,3,4,5,6,7,8$ et 9 , un des termes au moins de la série complémentaire sera augmenté d'une unité, et même plusieurs de ses termes pourront en même temps être augmentés d'une unité; car il en sera ainsi des entiers, que contiennent les multiples $n \cdot \frac{m}{n}, 2 n \cdot \frac{m}{n}, 3 n \cdot \frac{m}{n}$ etc., et ces entiers forment les termes de la série complémentaire $(\boldsymbol{A}$.). Par exemple, si la fraction $x_{\varepsilon-1}$ s'approche d'abord de la valeur $\frac{m}{n}=\frac{3}{5}$, l'entier que contient $5 x_{\varepsilon-1}$ est toujours encore $<3$, et par conséquent $=2$; mais aussitôt que $x_{e-1}$ devient égal à $\frac{3}{5}$, l'entier devient $=3$. Si $x_{\varepsilon-1}$ s'approche de la valeur $\frac{m}{n}=\frac{2}{3}$, les entiers que contiennent $3 x_{\varepsilon-1}, 6 x_{\varepsilon-1}$ et $9 x_{\varepsilon-1}$ sont $<2,<4$ et $<6$, et par conséquent $=1,3$ et 5 ; mais aussitôt que $x_{\varepsilon-1}$ devient égal à $\frac{2}{3}$, ou plutôt (une fraction décimale égale à $\frac{2}{3}$ n'existant pas): aussitôt que $x_{\varepsilon-1}$ a dépassé de la plus petite quantité possible la fraction $\frac{2}{3}$, les entiers que contiennent $3 x_{\varepsilon-1}, 6 x_{\varepsilon-1}$ et $9 x_{e-1}$ seront $=2,4$ et 6 . Donc, dans le premier 
exemple de $\frac{m}{n}=\frac{3}{5}$, un seul terme de la série complémentaire, celui de $5 x_{\varepsilon-1}$, a augmenté d'une unité; dans le second exemple de $\frac{m}{n}=\frac{2}{3}$, trois termes, ceux de $3 x_{\varepsilon-1}, 6 x_{\varepsilon-1}$ et $9 x_{\varepsilon-1}$, ont éprouvé cette augmentation.

C. D'un autre côté, aucun terme de la série complémentaire qui convient à $x_{\varepsilon-1}$, ne changera pas de valeur, quelle que soit la fraction irréductible $\frac{m}{n}$, par laquelle passe $x_{\varepsilon-1}$, si le dénominateur $n$ de cette fraction $\frac{m}{n}$ est plus grand que 9 ; car les entiers que contient un multiple quelconque de $x_{\varepsilon-1}$ ne peuvent alors augmenter d'une unité, à moins que ce multiple ne soit plus grand que $9 x_{\varepsilon-1}$, multiple dont jl ne s'agit pas ici. Par exemple, si $x_{\varepsilon-1}$ s'approche d'abord de $\frac{m}{n}=\frac{7}{11}$, les termes de la série complémentaire, c'est$\grave{a}-$ dire les entiers contenus dans $2 x_{\varepsilon-1}, 3 x_{\varepsilon-1}, 4 x_{\varepsilon-1}, 5 x_{\varepsilon-1}, 6 x_{\varepsilon-1}, 7 x_{\varepsilon-1}$, $8 x_{\varepsilon-1}$ et $9 x_{\varepsilon-1}$ seront $1,1,2,3,3,4,5$ et 5 , et ils continueront d'avoir ces valeurs, même après que $x_{\varepsilon-1}$ aura passé $\frac{7}{11}$; ce n'est que $11 x_{\varepsilon-1}$ qui, contenant 6 entiers, toutes les fois que $x_{\varepsilon-1}$ est $<\frac{7}{11}$, en prend 7 entiers, aussitôt que $x_{\varepsilon-1}$ a atteint ou dépassé la fraction $\frac{m}{n}=\frac{7}{11}$. En effêt, soit $x_{\varepsilon-1}=\frac{m}{n}+k$, où $k$ représente une fraction quelconque, on a $\mu x_{\varepsilon-1}=\frac{\mu m}{n}+\mu k$. Ici $\frac{\mu m}{n}$ est évidemment moindre que $m$ entiers, tant que $\mu<n$ : donc $\frac{\mu m}{n}$ ne contient que $m-1$ entiers, suivis d'une fraction $<1$, par ex. de la fraction $\lambda$. Or, $k$ pourra être supposé toujours si petit, que $\mu k+\lambda$ soit encore $<1$, donc aussi $\mu x_{\varepsilon-1}=\frac{\mu m}{n}+\mu k$ ne contiendra encore que $m-1$ entiers, et il existera par conséquent toujours encore des fractions $x_{\varepsilon-1}$ plus grandes que $\frac{m}{n}$ qui, de même que celles moindres que $\frac{m}{n}$, ne contiennent que $m-1$ entiers, tant que $\mu$ est moindre que $n$. Mais il en est autrement, aussitôt que $\mu$ devient $=n$; alors déjà $\mu x_{\varepsilon-1}$ est $=n \cdot \frac{m}{n}=m$, et déjà $\mu x_{\varepsilon-1}$ contient $m$ entiers, au lieu de $m-1$ entiers qui se trouvaient dans $\mu x_{\varepsilon-1}$, ou dans $n x_{\varepsilon-1}$, lorsque $x_{\varepsilon-1}$ était encore au dessous de $\frac{m}{n}$.

Donc aucun terme de la série complémentaire que donnẹnt les multiples $2 x_{\varepsilon-1}, 3 x_{\varepsilon-1}, 4 x_{\varepsilon-1}, 5 x_{\varepsilon-1}, 6 x_{\varepsilon-1}, 7 x_{\varepsilon-1}, 8 x_{\varepsilon-1}$ et $9 x_{\varepsilon-1}$ de $x_{\varepsilon-1}$ Crelle's Journal f. d. M. Bd. XXX. Heft 3. 
ne changera pas de valeur, à moins que $x_{\varepsilon}$ ne passe par une fraction irréductible $\frac{m}{n}$, dont le dénominateur est un des nombres $2,3,4,5,6,7,8,9$.

D. Il suit de là, que le nombre des changements qu'éprouvera la série complémentaire, sera égal au nombre des fractions irréductibles $\frac{m}{n}$, dont les dénominateurs sont les différents nombres $2,3,4,5,6,7,8$ et 9 .

Ces fractions sont les suivantes:

$$
\text { 1. }\left\{\begin{array}{llllll}
\frac{1}{9} & \frac{2}{9} & \frac{4}{9} & \frac{5}{9} & \frac{7}{9} & \frac{8}{9} \\
\frac{1}{8} & \frac{3}{8} & \frac{5}{8} & \frac{7}{8} & & \\
\frac{1}{7} & \frac{2}{7} & \frac{3}{7} & \frac{4}{7} & \frac{5}{7} & \frac{6}{7} \\
\frac{1}{6} & \frac{5}{6} & & & \\
\frac{1}{5} & \frac{2}{5} & \frac{3}{5} & \frac{4}{5} & \\
\frac{1}{4} & \frac{3}{4} & & & \\
\frac{1}{3} & \frac{2}{3} & & & \\
\frac{1}{2} & & & &
\end{array}\right.
$$

ou bien, rangées suivant leur valeurs,

2. $\frac{m}{n}=\frac{1}{9} \frac{1}{8} \frac{1}{7} \frac{1}{6} \frac{1}{5} \frac{2}{9} \frac{1}{4} \frac{2}{7} \frac{1}{3} \frac{3}{8} \frac{2}{5} \frac{3}{7} \frac{4}{9} \frac{1}{2} \frac{5}{9} \frac{4}{7} \frac{3}{5} \frac{5}{8} \frac{2}{3} \frac{5}{7} \frac{3}{4} \frac{7}{9} \frac{4}{5} \frac{5}{6} \frac{6}{7} \frac{7}{8} \frac{8}{9}$. Leur nombre est $=27$ : donc il y aura 27 changements de la série complémentaire, et par suite le nombre des différentes séries complémentaires elles mêrnes, qui existent, est $=28$; 'comme l'énonce le théorème.

E. Il sera maintenant facile de trouver par ce qui a été dit en (C.) les termes des différentes séries complémentaires.

D'abord la séríe complémentaire propre aux fractions $x_{\varepsilon-1}>0<\frac{1}{9}$ est évidemment $0, \theta, 0, \theta, 0,0,0,0$; car aucun des multiples $2 x_{\varepsilon-1}, 3 x_{\varepsilon-1}$, $4 x_{\varepsilon+1}, 5 x_{\varepsilon-1}, 6 x_{\varepsilon-12}, 7 x_{\varepsilon-1}, 8 x_{\varepsilon-1}$ et $9 x_{\varepsilon-1}$ ne contient ici un entier. Aussitôt que $x_{\varepsilon-1}$ a dépaşsé la valeur $\frac{1}{9}$, le multiple $9 x_{\varepsilon-1}$ de $x_{-1}$ contient une unité, les autres multiples n'en ont pas encore, jusqu'à $x_{\varepsilon-1} \neq \frac{1}{8}$; donc la série complémentaire propre aux fractions $x_{\varepsilon-1}>\frac{1}{9}<\frac{1}{8}$ est $0 ; 0,0 ; 0,0,0,0,1$. Si $x_{\varepsilon-1}$ a atteint la valeur $\frac{1}{8}$, le multiple $8 x_{\varepsilon-1}$ de $x_{\varepsilon-1}$ contient une unité, 
dans les autres multiples les nombres des entiers ne changent pas; donc la série complémentaire propre aux fractions $x_{\varepsilon-1}>\frac{1}{8}<\frac{1}{7}$ est $0,0,0,0,0,0,1,1$; et ainsi de suite. Généralement, chaque fois que $x_{\varepsilon-1}$ passe par une des fraclions $\frac{m}{n}$ (2.), ceux des multiples de $x_{\varepsilon-1}$, donc le nombre est, ou égal au dénominateur $n$ de la fraction, ou non-premier à $n(B$.), prendront un entier de plus. Suivant cette règle on trouvera les valeurs suivantes des termes des différentes séries complémentaires. Nous désignerons ces séries par $s$.

Les termes des sćries

- complémentaires conviendront aux fractions

pour les multiples $2 \mathbf{3} \mathbf{5} 8 \mathbf{8}$

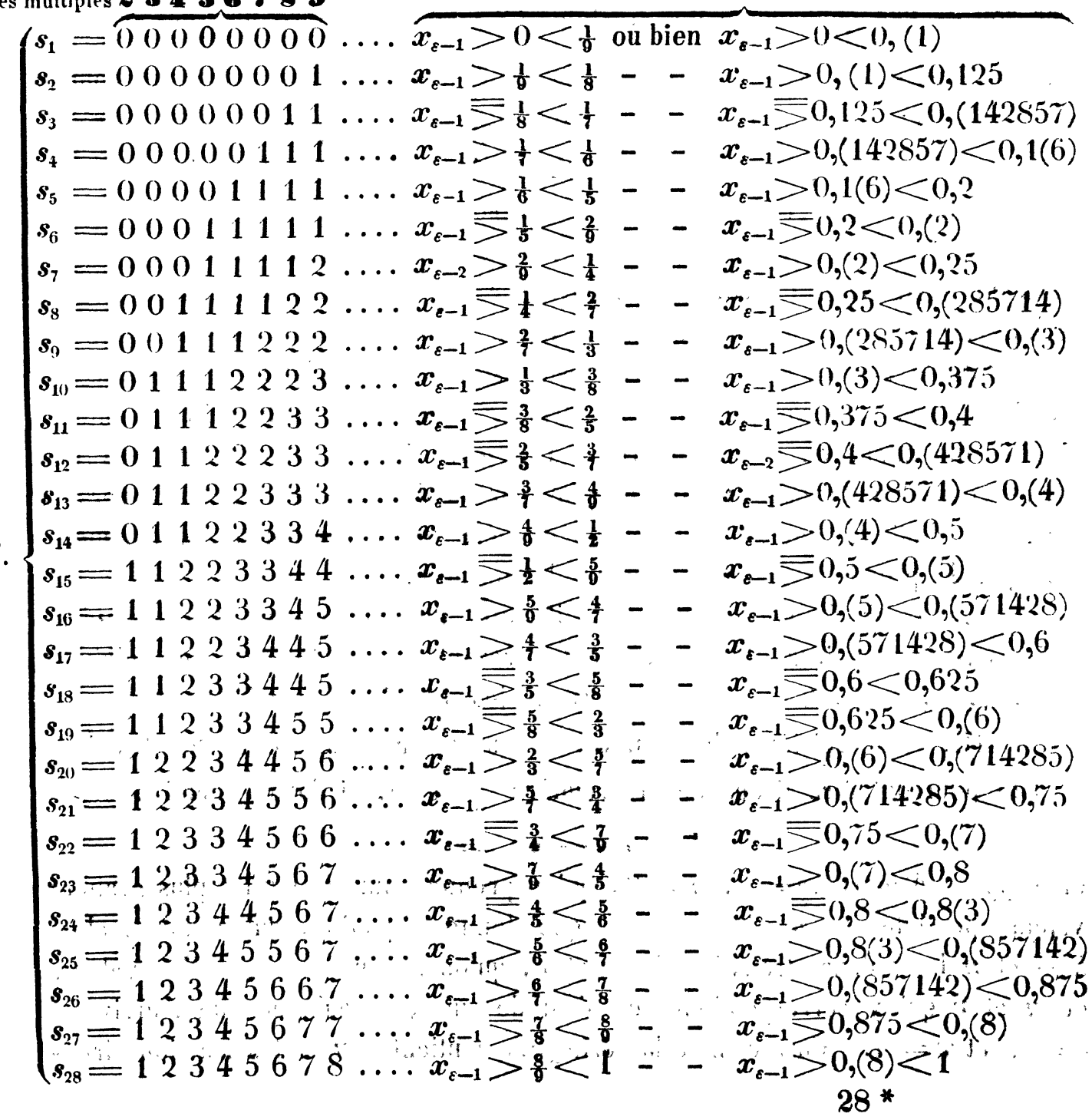


Dans les expressions des fractions en décimales, à droite de la table, les chiffres qui sont entre des crochets signifient des périodes, qui reviennent à l'infini; là où il n'y a pas des chiffres mis entre des crochets, les décimales expriment exacteinent la fraction dont il s'agit.

\section{Noles.}

I. Il est à remarquer que dans la série des fractions (2.), qui représentent les valeurs de $x_{\varepsilon-1}$ pour lesquelles les termes des séries complémentaires (3.) changent de valeur, la somme de chaque couple de fractions à égale distance du commencement et de la fin, est $=1$. Cela est clair en soi même; car soit $\frac{m}{n}$ une quelconque des fractions (2.), c'est-à-dire une des fractions irréductibles $<1,1-\frac{m}{n}=\frac{n-m}{n}$ en est évidemment une autre; et comme la série (2.) comprend toutes les fractions qui existent pour un dénominateur $<10$, il faut que pour chaque fraction, il s'en trouve dans la série une autre qui, ajoutée à la première, donne 1 pour somme.

II. Pareillement les termes de chaque série complémentaire (3.), à une distance quelconque du commencement, par ex. de la série $s_{\lambda}$, ajoutés aux termes de la série $s_{28-\lambda}$, qui est à égale distance de la fin, donnent les termes de la dernière série $s_{28-\lambda}$. Cela est également clair; car, comme les fractions (2.), en allant de gauche à droite, augmentent de la même manière, qu'elles diminuent en allant de droite à gauche, il faut, que les termes des séries complémentaires, en commençant par la dernière et en allant vers la première, diminuent aussi de la même manière qu'ils augmentent en commençant par la première et en allant vers la dernière.

En vertu de celte remarque, il suffit de calculer les 14 premières séries complémentaires; les autres se trouveront immédiatement, en déduisant de $1,2,3,4,5,6,7$ et 8 les termes des 14 séries calculées.

III. En troisième lieu, il est à remarquer, que toutes les fractions $\frac{m}{n}$ (2) pourront être tirées de la première $\frac{1}{9}$ seule, si dans l'équation

\section{4. $n u-m v=1$}

on suppose d'abord $m=1, n=9$, qu'on prenne pour $u$ et $v$ les plus grandes valeurs possibles de $u<v, v<10$ que donne l'équation, qu'on donne alors à $\boldsymbol{m}$ et $\boldsymbol{n}$ les valeurs trouvées de $\boldsymbol{u}$ et $\boldsymbol{v}$, qu'on prenne les nouvelles valeurs de $\dot{u}$ et $v$ qui y correspondent, et ainsi de suite, jusqu'à ce qu'on arrive à $u=1$ et $v=1$. Car, comme la série (2.) contient toules les fractions irré- 
ductibles qui existent, avec un dénominateur $<10$, il n'y a pas de fraction de cette sorte qui puisse tomber entre deux fractions consécutives de la série; donc chacune de deux fractions consécutives est toujours moins différente de l'autre, que ne l'est aucune autre fraction, dont le dénominateur n'est pas plus grand que 9. Or, cette propriété est effectivement celle de la fraction $\frac{u}{v}$ envers la fraction $\frac{m}{n}$, en donnant à $u$ et $v$ les plus grandes valeurs possibles et $<10$; car en développant $\frac{m}{n}$ en fraction continue, $\frac{u}{v}$ est alors la dernière des fractions convergentes que présente la fraction continue, et cette fraction convergente a la proprieté en question.

En faisant le calcul, on trouve ce qui suit.

L'équation (4.) donne
1. $\frac{m}{n}=\frac{1}{9}, \quad 9 u=v+1, u=1,2,3, \ldots$,
2. $\frac{m}{n}=\frac{1}{8}, \quad 8 u=v+1, \quad u=1,2,3, \ldots$. ,
3. $\frac{m}{n}=\frac{1}{7}, \quad 7 u=v+1, \quad u=1,2,3, \ldots$,
4. $\frac{m}{n}=\frac{1}{6}, \quad 6 u=v+1, \quad u=1,2,3, \ldots$,
5. $\frac{m}{n}=\frac{1}{5}, \quad 5 u=v+1, \quad u=1,2,3, \ldots$,
$v=8,17,26, \ldots$
Donc
6. $\frac{m}{n}=\frac{2}{9}, \quad 9 u=2 v+1, \quad u=1,3,5, \ldots .$.$$
v=7,15,22, \ldots,
$$$$
\frac{u}{v}=\frac{1}{8} \text {; }
$$$$
v=6,13,19, \ldots,
$$$$
\frac{u}{v}=\frac{1}{7}
$$$$
v=5,11,16, \ldots .
$$$$
\frac{u}{v}=\frac{1}{6} \text {; }
$$$$
v=4,9,14, \ldots,
$$$$
\frac{u}{v}=\frac{1}{5} \text {; }
$$$$
\frac{u}{v}=\frac{2}{9} \text {; }
$$$$
v=4,13,22, \ldots, \quad \frac{u}{v}=\frac{1}{4} ;
$$
7. $\frac{m}{n}=\frac{1}{4}, \quad 4 u=v+1, \quad u=1,2,3, \ldots .$.

$$
v=3,7,11, \ldots .
$$$$
\frac{u}{v}=\frac{2}{7} \text {; }
$$
8. $\frac{m}{n}=\frac{2}{7}, \quad 7 u=2 v+1, \quad u=1,3,5, \ldots$,

$$
v=3,10,17, \ldots, \quad \frac{u}{v}=\frac{1}{3} ;
$$
9. $\frac{m}{n}=\frac{1}{3}, \quad 3 u=v+1, \quad u=1,2,3,4, \ldots$.

$$
v=2,5,8,11, \ldots, \quad \frac{u}{v}=\frac{3}{8} ;
$$
10. $\frac{m}{n}=\frac{3}{8}, \quad 8 u=3 v+1, \quad u=2,5,8, \ldots$,
$v=5,13,21, \ldots, \quad \frac{u}{v}=\frac{2}{5} ;$
11. $\frac{m}{n}=\frac{2}{5}, \quad 5 u=2 v+1$,
$u=1,3,5 ; 7, \ldots \ldots$
$v=2,7,12,17, \ldots$.
$\frac{u}{v}=\frac{3}{7}$;
12. $\frac{m}{n}=\frac{3}{7}, \quad y u=3 v+1, \quad u=1,4,7, \ldots .$.

$$
v=2,9,16, \ldots, \quad \frac{u}{v}=\frac{4}{9} ;
$$
13. $\frac{m}{n}=\frac{4}{9}, \quad 9 u=4 v+1, \quad u=1,5,9, \ldots$.
$v=2,11,20, \ldots, \quad \frac{u}{v}=\frac{1}{2}$;
14. $\frac{m}{n}=\frac{1}{2}, \quad 2 u=0+1$
$u=1,2,3,4,5,6, \ldots, v=1,3,5,7,9,11, \ldots, \frac{u}{v}=\frac{5}{9}$

5 
Pour

Donc

15

$\underline{m}=\frac{5}{9}$

Donc

15. $\frac{m}{n}=\frac{5}{9}$

$9 u=5 v+1$

$u=4,9,13, \ldots$

$v=7,16,23, \ldots$

$\frac{u}{v}=\frac{4}{7}$;

16. $\frac{m}{n}=\frac{4}{7}$

$7 u=4 v+1$

$u=3,7,11, \ldots$

$v=5,12,19, \ldots$,

$\frac{u}{v}=\frac{3}{5}$;

17. $\frac{m}{n}=\frac{3}{5}$

$5 u=3 v+1$

$u=2,5,8, \ldots$.

$v=3,8,13, \ldots$

$\frac{u}{v}=\frac{5}{8}$;

18. $\frac{m}{n}=\frac{5}{8}$

$8 u=5 v+1$

$u=2,7,12, \ldots$,

$v=3,11,19, \ldots, \quad \frac{u}{v}=\frac{2}{3} ;$

19. $\frac{m}{n}=\frac{2}{3}$,

$3 u=2 v+1$

$u=1,3,5, \tau, \ldots$

$v=1,4,7,10, \ldots$

$\frac{u}{v}=\frac{5}{7}$;

20. $\frac{m}{n}=\frac{5}{7}$

$7 u=5 v+1$

$u=3,8,13, \ldots$,

$v=4,11,18, \ldots$,

$\frac{u}{v}=\frac{3}{4}$

5.

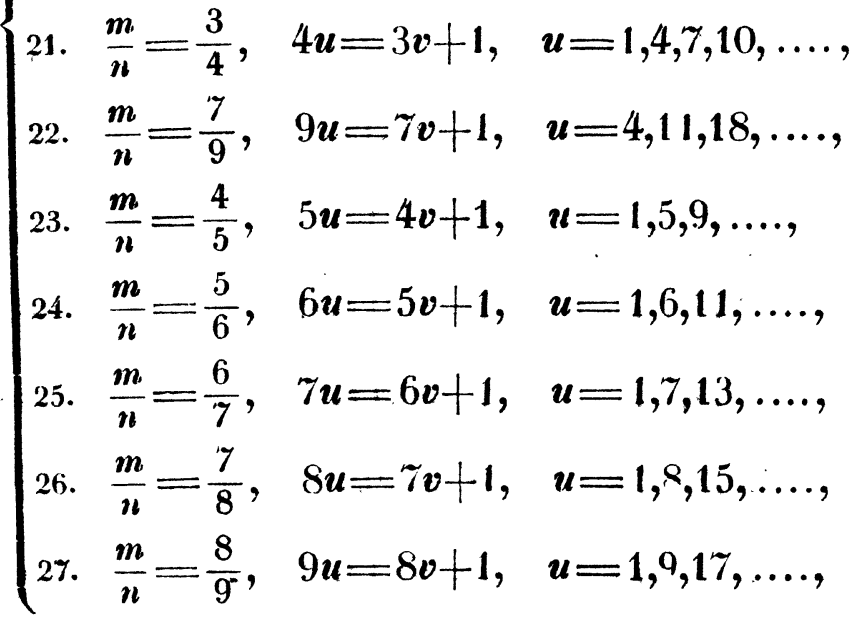

$v=1,5,9,13, \ldots, \quad \frac{u}{v}=\frac{7}{9}$;

$v=5,14,23, \ldots, \quad \cdot \frac{u}{v}=\frac{4}{5} ;$

$v=1,6,11, \ldots, \quad \frac{u}{v}=\frac{5}{6} ;$

$v=1,7,13, \ldots, \quad \frac{u}{v}=\frac{6}{7} ;$

$v=1,8,15, \ldots, \quad \frac{u}{v}=\frac{7}{8} ;$

$v=1,9,17, \ldots ., \quad \frac{u}{v}=\frac{8}{9} ;$

$v=1,10,19, \ldots, \quad \frac{u}{v}=\frac{1}{1}=1$.

Voilà toutess les fractions $\frac{u}{v}$ de la série (2.).

IV. Il est encore aisé de voir, comment tout ce qui a été dit jusqu'ici, et le théorème lui mème, pourrait être étendu aux cas, où il s'agissait d'un plus grand nombre de multiples du nombre proposé $Z$. Le nombre 27 des changements de la série complémentaire est celui de la somme des nombres premiers relatifs aux nombres $2,3,4,5,6,7,8,9$, comme on le peut voir dans le tableau (1.) des valeurs $\frac{m}{n}$ de $x_{\varepsilon \rightarrow t}$, poụ lesquelles les changements de la série complémentaire ont lieu. Donc, généralement, si l'on vouloit pousser les multiples de $x_{\varepsilon-1}$ non seulement jusqu'à $9 x_{\varepsilon-1}$, mais encore jusqu'à $\boldsymbol{\nu} x_{\varepsilon-1}$, le nombre des changements de la série qomplémẹlaire, qui aurolt alors $\nu-1$ termes, au lieu de 8 , serait égal à la somme des nombres premiers relatifs aux nombres $2,3,4,5, \ldots \ldots$, ot lo nombre des différentes séries complé- 
mentaires seroit plus grand d'une unité. Ce nombre est déjà très élevé pour la valeur 99 de $\nu$, valeur qui seroit propre à une application au calcul de chiffres.

\section{Application du théorème ci-dessus à la mulliplication de grands nombres.}

A. Pour trouver le produit d'un nombre quelconque douné $\boldsymbol{Z}$ par un autre nombre quelconque, sans multiplier successivement les chiffres de $\boldsymbol{Z}$ par les chiffres du multiplicateur, on aura besoin des multiples $2 \boldsymbol{Z}, 3 \mathbf{Z}, 4 \mathbf{Z}, 5 \mathbf{Z}$, (i $\mathbf{Z}, \boldsymbol{i} \mathbf{Z}, 8 \mathbf{Z}, 9 \mathbf{Z}$ du multiplicande $\boldsymbol{Z}$. Ces multiples connus, il n'y aura qu'à les copier, en les mettant aux places que le multiplicateur préscrit, et à les additionner.

B. Or, on aura ces multiples, écrits l'un sous l'autre, et les unités des différentes ordres, mises partout dans les mêmes lignes verticales; si l'on connait les séries des chiffres qui doivent ètre écrits dans ces différentes lignes.

La table qui se trouve à la fin de ce mémoire, donne immédiatement et sans calcul ces séries verticales de chiffres, et elle est fondée sur le théorème de Mr. Slonimsky. Voici comment.

C. Pour plus de clarté, prenons pour exemple le nombre $\boldsymbol{Z}=$ $5390 \$ 7611428992 \% 4$. Les multiples de ce nombre, qu'il s'agit de trouver sans calcul, sont les suivants:

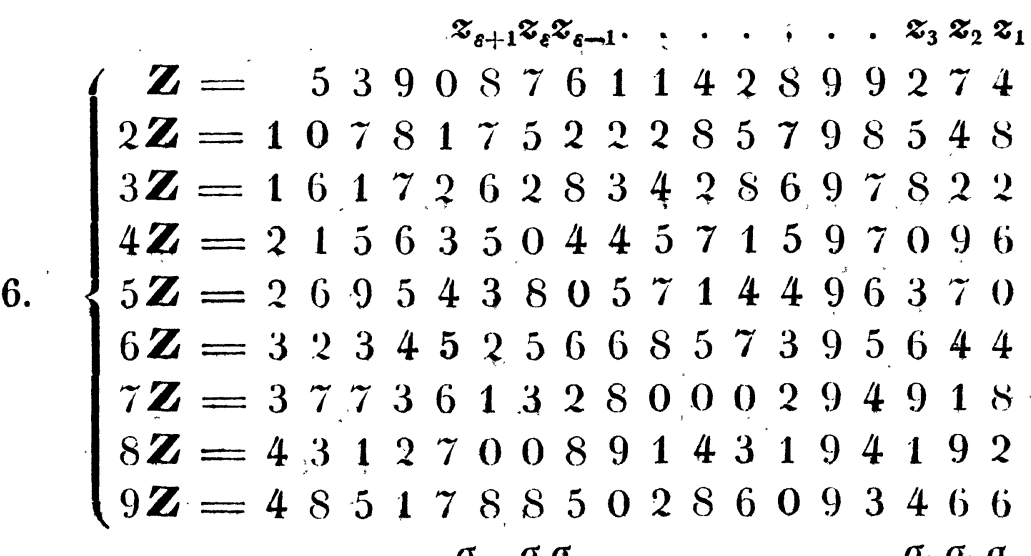

$$
\sigma_{s+1} \sigma_{\varepsilon} \sigma_{\varepsilon+1} \cdot \text {. . . . . } \sigma_{1} \sigma_{2} \sigma
$$

Nous désignerons par $\boldsymbol{z}_{\varepsilon+1}, \approx_{\varepsilon}, \boldsymbol{x}_{\varepsilon-1}$ trois chiffres quelconques cansécutifs du nombre $Z$, par $\sigma_{\varepsilon+1}, \sigma_{\varepsilon}, \sigma_{\varepsilon+1}$ les séries des chiffres dans les lignes verticales qui passent par les chiffres $\boldsymbol{x}_{\varepsilon+1}, z_{\varepsilon}$ et $\boldsymbol{z}_{\varepsilon-1}$, et par $x_{\varepsilon}, x_{\varepsilon-1}$ et $x_{\varepsilon-2}$ les fractions décimales $s_{q}$ formées par les chiffres de $\mathbb{Z}$ qui suivent les ehiffres $\boldsymbol{z}_{\varepsilon+1}$, $\boldsymbol{z}_{\varepsilon}$ et $\boldsymbol{x}_{\varepsilon+1}$. 
D. La série $\sigma_{\varepsilon-1}$ sera celle des seconds chiffres des multiples de $\boldsymbol{z}_{\varepsilon-1}$, augmentés de la série complémentaire qui, tirée de (3.), convient à la fraction $x_{\varepsilon-2}$; et les premiers chiffres de ces mêmes multiples, ainsi augmentés, ne seront autre chose que la série complémentaire qui convient à la fraction $x_{\varepsilon-1}$; les premiers chiffres des multiples, quels qu'ils soient, formeront toujours une quelconque des 28 séries complémentaires; car, suivant le théorème, il n'en existe point d'autres. Donc, aussitôt que l'on connaîtra la série complémentaire à ajouter aux multiples de $z_{\varepsilon-1}$, ces multiples augmentés seront connus, par conséquent aussi leurs premiers chiffres le seront, et par suite non seulement les chiffres de la série $\sigma_{\varepsilon-1}$, qui sont les seconds chiffres des multiples augmentés, mais aussi ceux de la nouvelle série complémentaire qui est à ajouter aux multiples du chiffre $z_{\varepsilon}$, qui dans $\boldsymbol{Z}$ précède $z_{\varepsilon-1}$, seront connus. Or, par là on aura aussi déjà obtenu les multiples de $z_{\varepsilon}$ augmentés de la nouvelle série complémentaire, et par conséquent non seulement les chiffres de la série $\sigma_{\varepsilon}$, qui sont les seconds chiffres de ces multiples augmentés, mais aussi la série complémentaire, formée par leurs premiers chiffres, qui est à ajouter aux multiples de $\boldsymbol{z}_{\varepsilon+1}$, et ainsi de suite.

Donc, aussitôt qu'on connaîtra la série complémentaire à ajouter aux multiples d'un quelconque des chiffes de $\boldsymbol{Z}$, par exemple du chiffre $z_{\lambda}$, non seulement la série $\sigma_{\lambda}$ sera connue, mais aussi toutes les autres séries $\sigma$, qui la précèdent, en seront obtenues successivement, et si $z_{\lambda}$ est le dernier des chiffres de $\boldsymbol{Z}$, toutes les séries $\sigma_{1}$ sans exception, pourront être trouvées, et le problème sera résolu.

Or la série complémentaire à ajouter aux multiples du dernier chiffre $z_{1}$ de $\mathbf{Z}$ est effectivement toujours connue; elle est $s_{1}=0,0,0,0,0,0,0,0$ (3.); car la fraction $x_{0}$, qui suit le dernier chiffre, est zéro. Donc le problème de trouver sans calcul les différentes séries $\sigma$, c'est-à-dire, les multiples de $\boldsymbol{Z}$, pourra être résolu aussitôt, qu'on uura calculé par avance les séries des seconds chiffres des multiples des différents nombres $0,1,2,3,4,5,6,7,8$ et !, augmentés de l'une ou de l'autre des 28 séries complémentaires, et qu'on aura indiqué le numéro des séries complémentaires formées dans chaque cas par les premiers chiffres de ces multiples; car, en premier lieu, les chiffres de $\boldsymbol{Z}$, quels qu'ils soient, se trouveront toujours parmi les nombres $0,1,2$, $3,4,5,6,7,8,9$; en second lieu, ce sera toujours une quelconque des 28 séries complémentaires qui est à ajouter aux multiples d'un chiffre quelconque $z_{\varepsilon-1}$ de $Z$; et en troisième lieu, chaque série complémentaire, ajoutée 
aux multiples du chiffre $\boldsymbol{z}_{\delta-1}$, donne la nouvelle série complémentaire à ajouter aux multiples du chiffre précédent $\approx_{\varepsilon}$; car, cette nouvelle série complémentaire est celle que forment les premiers chiffres des multiples du chiffe $z_{\varepsilon-1}$.

$\boldsymbol{E}$. Ce sont les résultats de ce calcul préliminaire, que présente la table à la fin du mémoire. Les 28 séries de nombres qui se trouvent dans la table au dessous de chacun des nombres $0,1,2,3,4,5,6,7,8,9$, écrits en gros caractères dans la première lignè horizontale, sont celles des seconds chiffres des multiples de ces nombres, augmentés des différentes séries complémentaires No. 1, 2, 3, ... jusqu'à 28, dont les numéros sont écrits dans les première et dernière lignes verticales de la table, et au côté droit de chacune de ses séries est indiqué le numéro de la série complémentairé que forment les premiers chiffres des multiples des nombres $0,1,2,3, \ldots .9$, augmentés de la série complémentaire dont le numéro est indiqué dans la première et la derniere colonne verticale.

F. Un seul exemple suffira, pour faire voir, comment la table a été calculée.

Soit proposé le chiffre $\%$.

Les multiples de ce nombre sont ..... $142128 \quad 3542 \quad 495663$ Ajoutons y, par exemple, la série complémen-

taire No. 19, qui, suivant (3.) est $\ldots \begin{array}{lllllllll}1 & 1 & 2 & 3 & 3 & 4 & 5 & 5\end{array}$

Les multiples de 7, augmentés de cetle série

complémentaire, sont . . . . . 15 22303845536168 Les seconds chiffres $5,2,1,8,5,3,1,8$ de ces multiples sont ceux qu'on trouve dans la table au dessous de 7 dans la $19^{\text {me }}$ ligne horizontule. Les premiers chiffres $1,2,3,3,4,5,6,6$ forment, suivant (3.), la série complémentaire No. 22, et ce numéro 22 est indiqué dans la table, immédiatement à la suite des seconds chiffres $5,2,0,8,5,3,1,8$.

C'est de cette manière que la table a été calculée. D'ailleurs elle présente encore devant les multiples $2 z, 3 z, 4 z, 5 z, 6 z, 7 z, 8 z, 9 z$ les chiffres de $0 \cdot z$ et $1 \cdot z$, et ces chiffres sont évidemment 0 et $z$ eux mêmes; car les termes correspondants des séries complémentaires sont toujours $=0$, parcequ'aucun des nombres $z=0,1,2,3,4,5,6,7,8,9$ n'a pas de premier chiffre.

$\boldsymbol{G}$. Faisons voir maintenant l'usage de la table, en l'appliquant à l'exemple (6.).

D'abord les multiples du premier chiffre $z_{1}=4$ de $Z$, à partir de la droite, sont $4,8,12,16,20,24,2 \&, 32,36$. Les seconds chiffes de ces 
multiples sont ceux de la série verticale $\sigma_{1}$ dans (6.); la table les présente sous 4 dans la première ligne horizontale. Les premiers chiffres $0,0,1,1$, $2,2,2,3,3$ des multiples de 4 forment, suivant (3.), la série complémentaire No. 12 , et ce numéro 12 se trouve indiqué dans la table à la suite de la série des seconds chiffres des multiples.

Les multiples du second chiffre $\mathfrak{x}_{2}=7$ de $z$ sont

$$
714212835424956 \quad 63
$$

Mais il faut $\mathrm{y}$ ajouter la série complé-

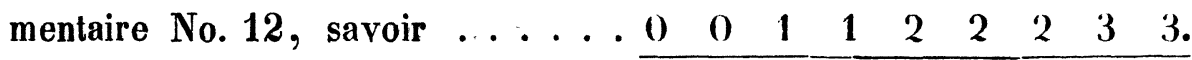

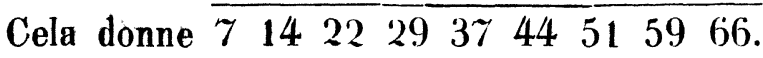

Les seconds chiffres $7,4,2,9,7,4,1,9,6$ de ces multiples sont ceux de la série $\sigma_{2}$ de (6.), et on les trouve dans la table, sous 7, dans la ligne horizontale No. 12. Les premiers chiffres $0,1,2,2,3,4,5,5,6$ des multiples forment, suivant (3.), la série complémentaire No. 21 , et ce numéro 21 se trouve indiqué dans la table à la suite des seconds chiffres.

Les multiples du troisième chiffre $z_{3}=2$ de $Z$ sont

$$
\begin{array}{llllllllll}
2 & 4 & 6 & 8 & 10 & 12 & 14 & 16 & 18 .
\end{array}
$$

Il faut $\mathrm{y}$ ajouter la série complémentaire

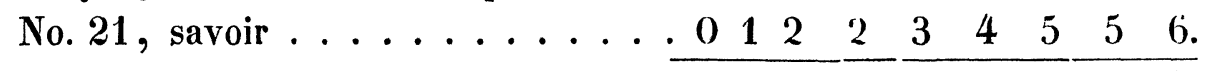

Cela donne $\begin{array}{llllllllll}5 & 5 & 10 & 13 & 16 & 19 & 21 & 24 .\end{array}$

Les seconds chiffres $2,5,8,0,3,6,9,1,4$ de ces multiples sont ceux de la série $\sigma_{3}$ de (6.), et on les trouve dans la table, sous 2 , dans la ligne horizontale No. 21 . Les premiers chiffres $0,(1,0,1,1,1,1,2,2$ des multiples forment, suivant (3.), la série complémentaire No. 8 , et ce numéro 8 est indiqué dans la table à la suite des seconds chiffres.

Et ainsi de suite.

$\boldsymbol{H}$. On voit par là, comment les multiples d'un nombre quelconque proposé $\boldsymbol{Z}$, peuvent être tirés de la table sans aucun calcul.

Dans notre exemple, on écrit d'abord les nombres que donne la table sous $4=z_{1}$ dans sa ligne horizontale No. 1 . Ces nombres sont ceux de la colonne $\sigma_{1}$ dans (6.). Puis, on écrit les nombres que donne la table sous $\tau=z_{2}$ dans la ligne horizontale No. 12, numéro indiqué à la suite des nombres qui formaient la série $\sigma_{1}$. Ce sont les termes de la colonne $\sigma_{2}$ dans (6.). Alors on écrit les nombres que donne la table sous $2=z_{3}$ dans la ligne horizontale No. 21, numéro indiqué 'à la suite des nombres de la série $\sigma_{2} ;$ et ainsi de suite. 
I. Maintenant, pour multiplier un nombre quelconque donné $\boldsymbol{Z}$ par un autre nombre quelconque $\boldsymbol{Y}$, le premier procédé qui s'offre seroit, d'écrire préalablement les multiples $1 \boldsymbol{Z}, 2 \boldsymbol{Z}, 3 \boldsymbol{Z}, 4 \boldsymbol{Z}, 5 \mathbf{Z}, 6 \mathbf{Z}, 7 \mathbf{Z}, 8 \boldsymbol{Z}, 9 \boldsymbol{Z}$ de $\boldsymbol{Z}$, tirés sans calcul de la table, comme il l'a été décrit en (H.); puis de mettre de la manière ordinaire, à leurs places, l'un sous l'autre, les multiples de $\boldsymbol{Z}$, comme le demandent les différents chiffres de $\boldsymbol{Y}$, et enfin, de prendre la somme de ces différentes lignes de nombres. Mais il n'est pas même nécessaire d'écrire préalablement les multiples de $\boldsymbol{Z}$; la table donne immédiatement les différentes lignes des nombres, dont on n'aura ensuite qu'à prendre la somme. Un exemple fera voir comment cela se pratique.

Soit le nombre ci-dessus

$$
\text { 7. }\left\{\begin{aligned}
\boldsymbol{Z} & =53908761142899274 \text { à multiplier } \\
\text { par } \boldsymbol{Y} & =495014320 \% 86
\end{aligned}\right.
$$

le calcul est le suivant:

$$
\begin{aligned}
& \boldsymbol{Z}=53908761142899274 \\
& \boldsymbol{Y}=\quad 495014320786
\end{aligned}
$$

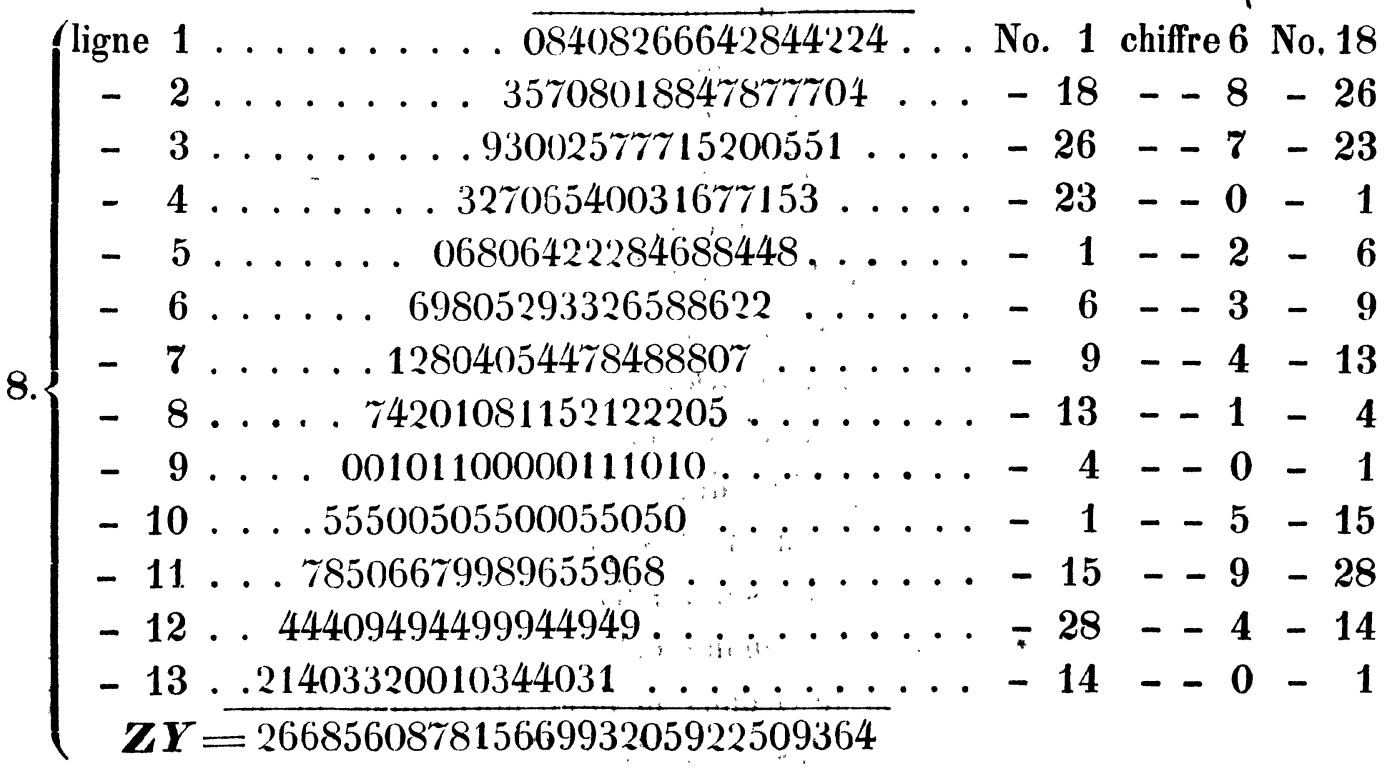

La ligne No. 1 du produit contient les seconds chiffres des différents multiples du dernier nombre 6 de $\boldsymbol{Y}$, que demandent les différents chiffres de $\boldsymbol{Z}$. La table les donne dans sa ligne horizontale No. 1 sous 6 ; par exemple, le premier nombre 5 de $\boldsymbol{Z}$ donne, suivant la table, le chiffre 0 ; le second nombre 3 donne le chiffre 8 ; le troisième nombre 9 donne le chiffre 4 etc. 
Puis les premiers chiffres des différents multiples du dernier nombre 6 de $\boldsymbol{Y}$ (si on les vouloit écrire), seroient tous à placer d'un pas de plus à gauche et aux mềnes places que prendront les seconds chiffres des multiples de l'avant-dernier chiffre 8 de $Y$. Ces premiers chiffres des multiples de 6 , dernier chiffre de $Y$, sont, suivant la table, compris tous dans la série complémentaire No.18, comme cela se trouve indiqué à la suite des seconds chiffres des multiples de 6 dans la ligne horizontale de la table No. 1.

Donc, en prenant maintenant les seconds chiffres des différents multiples du l'avant-dernier chiffre 8 de $Y$, et en $y$ ajoutant en même temps les premiers chiffres des multiples de 6 , qui ne sont pas encore écrits, on les trouvera tous dans la ligne horizontale No. 18 de la table sous 8; et de là sont tirés les chiffres de la ligne No. 2 dans (8.). En effet, le premier chiffre 5 de $Z$ donne en vertu de la ligne horizontale No. 18 de la table sous 8 le chiffre 3 , le second chiffre 3 de $\boldsymbol{Z}$ donne le chiffre 5 , le troisième chiffre 9 de $\boldsymbol{Z}$ donne le chiffre $\boldsymbol{Z}$ etc., et ce sont les chiffres de la ligne No. 2 dans (8.).

Pạr cela, on a maintenant introduit dans le produit à calculer les seconds et les premiers chiffres de tous les produits du dernier chiffre 6 de $\boldsymbol{Y}$, par les différents chiffres de $\boldsymbol{Z}$, et par conséquent tout ce qui concerne ces produits. Aussi a t'on déjà écrit les seconds chiffres des produits de l'avant-dernier chiffre 8 de $\boldsymbol{Y}$, par les différents chiffres de $\boldsymbol{Z}$; ils sont compris dans les nombres de la ligne No. 2 dans (8.). Mais les premiers chiffres de ces produits ne sont pas encore entrés en calcul.

Ces premiers chiffres, si on les vouloit écrire, seroient à placer tous à un pas de plus ̀̀ gauche, et aux mềnes places qui conviennent aux seconds chiffres des multiples du chiffre 7, qui dans $\boldsymbol{Y}$ précède l'avant-dernier chiffre 8. Mais ces premiers chiffres des multiples de 8, avant-dernier chiffre de $\boldsymbol{Y}$, multiples qui sont déjà augmentés par les preıniers chiffres des multiples du dernier chiffre 6 de $\boldsymbol{Y}$, sont, suivant la table, compris tous dans la série complémentuire No 26 ; comme cela se trouve indiqué à la suite des seconds chiffres des multiples de 8 dans la ligne horizontale de la table No. 18. Donc, en prenant maintenant les seconds chiffres des différents multiples du chiffre 7 , qui dans $\boldsymbol{Y}$ précède l'avant-dernier chiffre 8 , et en $\mathrm{y}$ ajoutant en même temps les premiers chiffres dés multiples de 8 , qui ne sont pas encore écrits, on les trouvera tous dans la ligne horizontale No. 26 de la table, sous 7, et c'est de là, que sont tirés les chiffres de la ligne No. 3 dans (8.). Le premier chiffre 5 de $\boldsymbol{Z}$ donne en vertu de la ligne horizontale No. 26 de la 
table, sous 7 , le chiffre 9 , le second chiffre 3 de $\boldsymbol{Z}$ donne le chiffre 3 , le troisième chiffre 9 de $\boldsymbol{Z}$ donne le chiffre 0 etc.

En continuant de cette manière, et conformément à ce qui se trouve annoté à la droite du calcul (8.), on tirera immédiatement de la table, et sans aucun calcul, les chiffres de toutes les lignes No. 1, 2, 3, 4 etc. dans (8.). Mais il s'agit encore de la dernière ligne No. 13. Elle contient les premiers chiffres des multiples du premier chiffre 4 de $\boldsymbol{Y}$, multiples, qui sont sous l'influence de la série complémentaire No. 14. Donc on les trouvera dans la ligne horizontale No. 14 de la table, sous 0 ; car le chiffre, qui dans $\boldsymbol{Y}$ précède le premier chiffre 4 , est zéro. Le premier chiffre 5 de $Z$ y donne le chiffre 2 , le second chiffre 3 donne le chiffre 1 , le troisième chiffre 9 donne le chiffre 4 etc.

Ayant tiré de la table les différentes lignes horizontales du produit $\mathbf{Z} \mathbf{Y}$, il ne reste qu'à en prendre la somme.

$K$. Si une seule personne faisait le calcul, en se servant de la table, l'opération seroit évidemment plus laborieuse encore, et le résultat plus sujet à des erreurs, que la multiplication ordinaire; mais il est clair, qu'aussitôt que deux personnes, dont l'une tire les chiffres de la table et les dicte à l'autre, qui les écrit, se réunissent, le calcul sera beaucoup moins fatiguant, et le résultat beaucoup plus sùr, que la multiplication ordinaire; car il n'y a ici rien à calculer, il n'y a qu'à lire, de vive voix, et à écrire. Donc, dans ce cas la table peut être effectivement d'une véritable et grande utilité pour la pratique. On pourroit même dire, que les produits, surtout de très-grands nombres, pourront être trouvés de cette manière plus facilement encore, et incontestablement avec plus de surété, que par les logarithmes.

Donc, déjà à cause de cette application du théorème de Mr. Slonimsky, ce thèorème est important; mais il peut bien outre cela être encore intéressant dans la théorie de nombres, et $\mathrm{y}$ avoir plusieurs autres conséquences.

Berlin au mois de Novembre 1845. 